\title{
Ambiente de aprendizaje en el hogar, instrucción materna y desempeño numérico temprano
}

\author{
Gina Bojorque $^{1}$ (D), Patricio Cabrera ${ }^{2}$ \\ ${ }^{1}$ Facultad de Filosofía, Universidad de Cuenca, Cuenca, Ecuador. \\ 2 AiA de Cabrera y Andrade Cía. Ltda.
}

Autor para correspondencia: gina.bojorque@ucuenca.edu.ec

Fecha de recepción: 3 de noviembre 2017 - Fecha de aceptación: 14 de diciembre 2017

\section{RESUMEN}

Incluso antes de iniciar la educación formal, existen grandes diferencias individuales en el desempeño numérico de los niños, sin embargo, el conocimiento sobre los mecanismos a través de los cuales los niños pequeños desarrollan habilidades numéricas es limitado. Ciertas características contextuales que rodean a los niños, incluyendo el ambiente de aprendizaje en el hogar y nivel de instrucción materna, han evidenciado relación con su desempeño numérico. Investigaciones sobre este tema se han llevado a cabo mayoritariamente en países desarrollados, lo cual implica que la generalización de hallazgos previos, sea una pregunta abierta para países en vías de desarrollo. El objetivo del presente estudio fue examinar la relación que existe entre el entorno de aprendizaje en el hogar de los niños y el nivel de instrucción materna con el desempeño numérico de los niños de primer año de básica. En el estudio participaron 176 niños de primer año de básica, de escuelas públicas y privadas de Cuenca. Los resultados indican que ciertas características del ambiente de aprendizaje en el hogar, como las actividades numéricas y de lectoescritura que los padres realizan con sus hijos, no se relacionan con el desempeño numérico de los niños. Sin embargo, el número de libros para niños disponibles en el hogar se relaciona predictivamente con su desempeño numérico. De igual manera, el nivel de instrucción materna presenta una relación predictiva con el desempeño numérico de los niños.

Palabras clave: Experiencias en el hogar, instrucción materna, habilidades numéricas.

\begin{abstract}
Even before the start of formal schooling, there are large individual differences in children's numerical performance, however, the knowledge about the mechanisms through which young children develop numerical abilities is limited. Certain contextual characteristics surrounding the children, including the home learning environment and the mother's education, have been associated with children's numerical performance. Research on this topic has been conducted mostly in developed countries, which makes the generality of those findings for developing countries, an open question. The aim of the present study was to examine the relations between the home learning environment and the mother's education and the numerical abilities of first-grade children. A total of 176 cuencano children from public and private schools participated in the study. The results indicate that certain characteristics of the home environment, such as the numerical and literacy activities that parents perform with their children, are not related to the numerical performance of the children. However, the number of books for children available in the home is predictively related to their numerical performance. Similarly, the level of maternal education has a predictive relationship with children's numerical performance.
\end{abstract}

Keywords: Home experiences, mother's instruction, numerical abilities. 


\section{INTRODUCCIÓN}

Las habilidades numéricas que poseen los niños pequeños son importantes para su desarrollo matemático futuro (Geary, 2011). Sin embargo, estudios previos indican que incluso antes de iniciar la educación formal ya existen grandes diferencias individuales en el desempeño numérico de niños (Anders, Grosse, Rossbach, Ebert, \& Weinert, 2013; Duncan, Dowsett, Claessens, Magnuson, Huston et al., 2007; Ramani \& Siegler, 2011). Aunque el conocimiento actual acerca de los mecanismos a través de los cuales los niños desarrollan las habilidades numéricas tempranas es limitado (Missall, Hojnoski, Caskie, \& Repasky, 2015), en general, las diferencias observadas en el desempeño numérico de los niños han sido relacionadas con sus características cognitivas, incluyendo memoria de trabajo e inteligencia (Geary, Hoard, \& Nugent, 2012; Swanson, Jerman, \& Zheng, 2008), o con características de su contexto, incluyendo la calidad del ambiente de aprendizaje en el hogar y el nivel de instrucción de la madre (Anders et al., 2012; Clements \& Sarama, 2008; Krajewski \& Schneider, 2009; Lefevre, Kwarchuk, Smith-Chant, Fast, Kamawar et al., 2009; Locuniak, Jordan, Kaplan, \& Ola, 2006). El presente estudio se enfocará exclusivamente en las características contextuales previamente mencionadas. En los párrafos que siguen, se discute en primer lugar, los resultados de investigaciones internacionales sobre la influencia de la calidad del ambiente de aprendizaje en el hogar en el desarrollo numérico de los niños. A continuación, se resumen los hallazgos de estudios sobre la influencia del nivel de instrucción materna en el desempeño numérico de los niños. Finalmente, se formula el objetivo del estudio y las preguntas de investigación.

\subsection{Ambiente de aprendizaje en el hogar y desempeño numérico}

La calidad del ambiente de aprendizaje en el hogar ha sido definida tanto por la naturaleza y frecuencia de las actividades que los padres realizan con sus hijos, por ejemplo, leer, jugar empleando números o contar; así como por la disponibilidad de recursos educativos que existen en casa, por ejemplo, el número de libros disponibles para niños (Anders et al., 2012; Melhuish, Phan, Sylva, Sammons, SirajBlatchford et al., 2008). En relación al primer aspecto, es decir, la naturaleza y frecuencia de las actividades que los padres realizan con sus hijos, se ha observado que los resultados de investigaciones previas han sido inconsistentes. Por un lado, algunos estudios han reportado que la calidad del ambiente de aprendizaje en el hogar a edades tempranas predice el desempeño matemático posterior (Anders et al., 2012; Krajewski \& Schneider, 2009). Más específicamente, se ha reportado que la frecuencia de actividades numéricas y de lectoescritura que realizan los padres con sus hijos se relaciona positivamente con el desempeño matemático de los niños (LeFevre, Polyzoi, Skwarchuk, Fast, \& Sowinski, 2010; Lefevre et al., 2009). Por ejemplo, LeFrevre et al. (2009) encontraron que las experiencias numéricas en el hogar reportadas por padres canadienses cuando sus hijos iniciaban el preescolar se correlacionaron positivamente con su desempeño numérico en primer y segundo grado ${ }^{1}$. De la misma manera, se ha encontrado que existe una relación positiva entre los procesos de lectoescritura y el desempeño numérico de los niños. En un estudio realizado en Inglaterra con niños de cinco años de edad, se halló que la conciencia fonológica, el vocabulario y el razonamiento no verbal predijeron el logro aritmético de estos (Simmons, Singleton, \& Horne, 2008), probablemente debido a que la adquisición de la aritmética está relacionada con el sistema de lenguaje (Dehaene, Piazza, Pinel, \& Cohen, 2003). Por el contrario, existen estudios que han encontrado que la frecuencia de actividades padre-hijo en el hogar, no se relaciona con el desempeño matemático de los niños (Blevins-Knabe \& Musun-Miller, 1996; Missall et al., 2015). Por ejemplo, en un estudio realizado con 72 padres estadounidenses, estos autores reportaron que no hubo una relación estadísticamente significativa entre el desempeño matemático de los niños de preescolar y las actividades matemáticas realizadas con sus padres en el hogar.

A pesar de la inconsistencia en los resultados de estos estudios, todos ellos reportan que existe gran variabilidad en la frecuencia con la que los padres realizan actividades con sus hijos en el hogar, así como en la clase de actividades que realizan. Por ejemplo, LeFevre et al. (2009) encontraron en una muestra de 258 padres canadienses que la gran mayoría jamás jugó con números imantados en la nevera.

1 El primero y segundo grado corresponden a segundo y tercero de básica en nuestro país. 
Adicionalmente los padres en este estudio indicaron que realizaban con menor frecuencia actividades numéricas que actividades de lectoescritura. Este resultado concuerda con los resultados de BlevinsKnabe \& Musun-Miller (1996) y de Skwarchuk (2009) quienes reportaron que la frecuencia de actividades de lectoescritura en el hogar era mayor que la frecuencia de actividades matemáticas.

En relación al segundo aspecto, es decir, la disponibilidad de recursos educativos en el hogar, más específicamente el número de libros disponibles para los niños, un estudio realizado por Evans, Kelley, \& Sikora (2014) que incluyó un total de 42 naciones que participaron en el Programa para la Evaluación Internacional de Estudiantes (PISA por sus siglas en inglés), reportó que la cantidad de libros en el hogar tiene una relación estadísticamente significativa con el desempeño académico de los estudiantes en todo el mundo. Dentro de este desempeño académico está incluida el área matemática. Estos autores observaron que esta relación se encontró de manera constante tanto en las naciones ricas como en las pobres, y tanto en Asia como en Europa y en América. Finalmente, el estudio de Evans y colegas (2014) encontró que las ganancias en el desempeño académico de los niños, debido al número de libros en el hogar, son mayores en los niveles menos favorecidos pues los libros benefician especialmente a niños pertenecientes a familias con poca educación y con empleos de bajo estatus. Estos autores concluyeron que cada libro adicional tiene un mayor impacto en el rendimiento de alguien que solo tiene una pequeña biblioteca hogareña que en el desempeño de alguien de una casa rebosante de libros. Así pues, el segundo libro y el tercer libro tienen un impacto mucho mayor que el libro 102 o el 103. Más específicamente, dentro del área de la matemática, Anderson, Anderson, \& Shapiro (2005) reportaron que el acceso a libros en el hogar es un medio importante para facilitar el aprendizaje de conceptos y procedimientos matemáticos de los niños. Una razón muy importante por la cual leer libros puede ayudar a los niños a aprender matemáticas, tiene que ver con el contexto significativo de las historias incluidas en los libros infantiles, los cuales generalmente son ilustrados. Así, Van den HeuvelPanhuizen \& Elia (2012) señalan que las matemáticas que los niños pueden encontrar en los libros ilustrados deben verse en un sentido amplio, lo cual significa que además de los temas matemáticos habituales como el número, la medición y la geometría, los temas relacionados con las matemáticas, por ejemplo, el crecimiento, los patrones, la equidad y la causa-efecto, también son considerados contenidos matemáticos.

\subsection{Instrucción de la madre y desempeño numérico}

Diferentes estudios internacionales han reportado que el nivel de educación de los padres tiene un efecto significativo en el desarrollo de habilidades numéricas de sus hijos (Aunio \& Niemivirta, 2010). Específicamente, el nivel de instrucción de la madre tiene una influencia significativa en la adquisición de habilidades numéricas tempranas (Anders et al., 2012) y es predictor del desempeño académico de sus hijos (Melhuish et al., 2008). Interesantemente, el nivel de instrucción materna se considera un factor que representa el nivel socioeconómico de la familia. Según Aunio \& Niemvirta (2010), a medida que aumenta el nivel de instrucción de la madre, es más probable que sus hijos disfruten de mejores condiciones socioeconómicas. Estos autores sugieren que la educación de los padres se correlaciona positivamente con el desempeño matemático de los niños en la escuela. Más aún, la existencia de una brecha socioeconómica en el desempeño matemático de los niños durante la infancia y los primeros años de escolaridad está bien documentada. Así, se ha encontrado que los niños de niveles socioeconómicos desfavorecidos tienen conocimientos y habilidades matemáticas más débiles que sus pares de niveles socioeconómicos medios (Clements \& Sarama, 2011; Jordan, Kaplan, Ramineni, \& Locuniak, 2009; Siegler \& Ramani, 2008; Starkey, Klein, \& Wakeley, 2004). Estas diferencias en la adquisición de habilidades numéricas tempranas son de gran preocupación, ya que los niños con menor desempeño corren el riesgo de continuar desempeñándose pobremente durante la escolarización formal (Jordan et al., 2009). Siegler \& Ramani (2009) ha propuesto que las diferencias en el conocimiento matemático reflejan diferencias en el apoyo ambiental para el aprendizaje, favoreciendo a los niños de entornos más opulentos. Así, los padres de ingresos medios participan en una gama más amplia de actividades matemáticas explícitas con sus hijos y lo hacen con mayor frecuencia que los padres de bajos ingresos (Clements \& Sarama, 2007; Starkey et al., 2004). 


\section{PRESENTE ESTUDIO}

Como se indicó anteriormente, desde temprana edad, existen diferencias significativas en el desempeño numérico de los niños, por lo que es importante aumentar nuestro entendimiento sobre los mecanismos que subyacen al desarrollo numérico temprano de los niños. Los resultados de estudios previos sobre la influencia de la estimulación numérica y de lectoescritura en el hogar en el desempeño numérico han sido inconsistentes, por lo que se requieren de estudios adicionales. Más aún si se considera que los estudios mencionados anteriormente se han realizado principalmente en Europa y Norteamérica y, debido a las diferencias culturales y educativas entre los países europeos, norteamericanos y sudamericanos, los resultados de estos estudios no pueden generalizarse a un contexto latinoamericano, limitando nuestra comprensión acerca de los mecanismos que subyacen al desarrollo numérico de los niños ecuatorianos. Por lo expuesto, el objetivo del presente estudio fue aportar a la literatura existente sobre el tema al explorar la relación existente entre el ambiente de aprendizaje en el hogar, el nivel de instrucción materna, y el desempeño numérico de los niños cuencanos al finalizar el primer año de básica. Para ello nos planteamos cinco preguntas de investigación:

1. ¿Existe relación entre las experiencias numéricas que los niños reciben en el hogar y su desempeño numérico al finalizar el primer año de básica?

2. ¿Existe relación entre las experiencias de lectoescritura que los niños reciben en el hogar y su desempeño numérico al finalizar el primer año de básica?

3. ¿Existe relación entre el número de libros disponibles para niños en el hogar y su desempeño numérico al finalizar el primer año de básica?

4. ¿Existe relación entre el nivel de instrucción materna y el desempeño numérico de los niños al finalizar el primer año de básica?

5. ¿Varía, según el género de sus hijos, la frecuencia con la que los padres reportan realizar actividades numéricas y de lectoescritura en el hogar?

\section{MÉTODO}

\subsection{Participantes}

Los participantes fueron 176 niños (90 varones y 87 mujeres) cerca de culminar el primer año de educación general básica. Los niños fueron seleccionados de nueve instituciones educativas de la ciudad de Cuenca, Ecuador. Para garantizar la representatividad de la muestra se incluyeron en el estudio escuelas públicas urbanas (3 escuelas), públicas rurales (3 escuelas) y privadas (3 escuelas). Se realizó una muestra aleatoria de los grupos de niños a evaluarse, incluyendo alrededor de 20 niños por institución. El promedio de edad de los niños fue de 6 años, 1 mes ( $D E=3.6$ meses). Previo al estudio se obtuvo el consentimiento de los padres de cada uno de los niños participantes.

La información sobre el nivel de instrucción materna fue proporcionada por las madres al inicio del año lectivo. Se establecieron en nueve categorías del nivel de instrucción materna: (1) sin educación; (2) básica elemental; (3) básica media; (4) básica superior; (5) bachillerato; (6) tercer nivel (tecnólogo); (7) tercer nivel (universidad); (8) maestría; y (9) doctorado. En nuestra muestra, ninguna madre registró un nivel de doctorado.

\subsection{Materiales}

Cuestionario para padres

Para evaluar las experiencias numéricas y de lectoescritura que los niños reciben en sus hogares, los padres completaron la versión en español del Cuestionario para Padres (Lefevre et al., 2009; Van Hoof, Bojorque, Torbeyns, Van Nijlen \& Verschaffel, en revisión). Este cuestionario consta de cuatro partes. 
La primera parte, con 17 ítems, evalúa el grado en el que los padres participan en actividades numéricas ${ }^{2}$ junto con sus hijos. Ejemplos de estos ítems son: "Escribir numerales”, "Jugar cartas”, o "Hablar sobre dinero cuando están de compras". La consistencia interna de esta parte del cuestionario para nuestra muestra (Alfa de Cronbach) fue de .86, lo que indica una buena confiabilidad. La segunda parte evalúa actividades de lectoescritura que realizan los padres junto con sus hijos. Esta parte consta de tres ítems: (1) "Escribir las letras del alfabeto", (2) "Identificar los sonidos de las letras del alfabeto", y (3) "Identificar los nombres de las letras escritas del alfabeto". La consistencia interna de esta parte del cuestionario para nuestra muestra (Alfa de Cronbach) fue de .64, indicando un nivel de confiablidad medio. La tercera parte, consiste en una pregunta sobre el número de libros en el hogar. Esta pregunta es: "Estime el número de libros para niños que hay en el hogar”. La cuarta parte evalúa las Actitudes de los padres hacía el número y la lectoescritura. Esta parte no fue incluida en el presente estudio.

Con excepción de las preguntas sobre el número de libros en el hogar, el cuestionario pregunta a los padres: “¿Con qué frecuencia usted y su hijo realizan las siguientes actividades? Los padres responden cada uno de los ítems empleando una escala de Likert de cinco puntos (0 - 4). El valor mínimo 0 indica que la actividad no ocurrió, 1 que la actividad ocurrió menos de una vez por semana, pero pocas veces al mes (de 1 a 3 veces), 2 que la actividad ocurrió una vez por semana, 3 que la actividad ocurrió algunas veces a la semana (de 2 a 4 veces), y 4 que la actividad ocurrió casi a diario.

\section{Test de evaluación numérica y aritmética}

Para evaluar las habilidades numéricas de los niños, se administró el Test de Evaluación Numérica y Aritmética (TENA) (Bojorque et al., 2015). Este test fue desarrollado en base a las destrezas propuestas en el currículo nacional para primer año de básica. La prueba está compuesta por 54 ítems divididos en nueve subescalas, (1) cuantificadores, (2) correspondencia uno a uno, (3) relaciones de orden más que / menos que, (4) conteo, (5) identificación de cantidades y asociación con numerales, (6) orden, (7) lectura y escritura de numerales, (8) suma, y (9) resta. Cada subescala consta de seis ítems ordenados según un nivel progresivo de complejidad. La prueba tiene dos partes: una parte individual con 29 ítems y una parte colectiva con 25 ítems. Los puntos se anotan de forma dicotómica, con una puntuación de 1 que indica una respuesta correcta y una puntuación de 0 una incorrecta (puntaje máximo $=54$ ). El Alfa de Cronbach de las puntuaciones de TENA para la muestra del presente estudio fue de .89. Para garantizar la concordancia entre evaluadores en la calificación del test se calculó el coeficiente de Kappa, con el $10 \%$ de la muestra, resultando en un nivel alto de confiabilidad entre evaluadores $K=$ $.95, p<.001$.

\subsection{Procedimiento}

Los padres recibieron el cuestionario y la respectiva explicación sobre cómo llenarlo, durante una reunión organizada en cada institución educativa en el séptimo mes del año escolar (marzo). Después de la reunión, los padres llenaron el cuestionario en sus hogares y lo devolvieron en el transcurso de una semana a cada maestra del grado. Se recolectó el 100\% de los cuestionarios. El 78\% de los cuestionarios fue completado por la madre de los niños, el 10\% por los padres, el 3\% lo llenaron juntos el padre y la madre, y el restante 9\% no indicó su relación con el niño. Por otro lado, los niños fueron evaluados mediante el TENA en el último mes del año escolar (junio). Todos los niños recibieron primero la parte colectiva del test en un aula silenciosa de su escuela, por un tiempo de 15 a 20 minutos. Posteriormente se les aplicó la parte colectiva en sus aulas de clase, con una duración de 30 a 40 minutos.

\footnotetext{
${ }^{2}$ Aunque el cuestionario se subdivide en 4 factores, para efectos de este estudio se consideró el puntaje global en los 17 ítems ya que solamente uno de ellos "contar en forma descendente" se correlacionaba con las habilidades numéricas de los niños”.
} 


\section{ANÁLISIS DE DATOS}

Todos los análisis estadísticos, descriptivos e inferenciales, se realizaron a través del software IBM SPSS Statistics, versión 22.0. Para cada una de las variables bajo estudio (es decir, actividades numéricas, actividades de lectoescritura, nivel de instrucción materna y TENA) se realizó la prueba de Kolmogorov-Smirnov determinando que todas las variables a excepción de las actividades numéricas no presentan una distribución normal. Por ello, para determinar la relación entre las variables se empleó la correlación de Spearman (Pedhazur, 1997). Por otra parte, para ilustrar la asociación encontrada entre el número de libros para niños, el nivel de instrucción materna y el desempeño numérico de los niños (medido a través del TENA), se empleó la prueba ANOVA con análisis post hoc de Tukey. Las variables que presentaron correlaciones significativas con el TENA (número de libros para niños y el nivel de instrucción materna) fueron agrupadas en cuatro partes porcentualmente iguales con cortes en tres cuartiles, para disponer de valores más informativos debido a que los datos no siguen una distribución normal (Rendón-Macías, Villasís-Keever, \& Miranda-Novales, 2016). Estos cuatro grupos homogéneos se consideraron variables predictores para un modelo de regresión lineal múltiple.

\section{RESULTADOS}

En primer lugar, se presentan lo estadísticos descriptivos sobre las diferentes variables bajo estudio: actividades numéricas, actividades de lectoescritura, número de libros para niños, nivel de instrucción materna, y desempeño numérico. A continuación, se presentan las correlaciones entre cada una de estas variables, seguidas del análisis de regresión lineal múltiple para predecir el desempeño numérico de los niños al final del año lectivo. Finalmente, se exponen los resultados sobre diferencias de género en la frecuencia de actividades numéricas y de lectoescritura que los niños reciben en el hogar.

\subsection{Análisis descriptivo}

En la Tabla 1 se presentan los estadísticos descriptivos de las actividades numéricas y las actividades de lectoescritura en el hogar, el número de libros para niños disponibles en casa, el nivel de instrucción materna, y el desempeño numérico de los niños. Como se puede observar en la Tabla 1, en primer lugar, se encontró que existe gran variabilidad entre padres en el reporte de la frecuencia de actividades específicas tanto numéricas como de lectoescritura que realizan con sus hijos. Así, se observó que existen padres que realizan estas actividades con sus hijos de manera frecuente mientras que otros lo realizan rara vez, e incluso hay padres que reportan que nunca lo han hecho. Más específicamente, en la Tabla 2, se observa que las actividades numéricas más frecuentes que se realizan en los hogares son contar objetos y escribir numerales, mientras que las actividades que menos se realizan son medir ingredientes al cocinar, utilizar un reloj, y utilizar el calendario. En relación a las actividades de lectoescritura, la actividad más frecuente reportada es escribir las letras, seguida de identificar sonidos del alfabeto y, por último, el identificar los nombres de las letras escritas del alfabeto. En segundo lugar, se encontró que la frecuencia de actividades numéricas padre-hijo es significativamente menor que la frecuencia de actividades de lectoescritura padre-hijo $F(352)=-2.72, p<0.01$. Es decir, los padres tienden a enfocarse más en realizar actividades de lectoescritura con sus hijos que en realizar actividades numéricas. En tercer lugar, el número de libros que disponen en el hogar, es altamente variable entre las familias. Así, se encontró que algunas familias carecen por completo de libros infantiles, mientras que otras reportan tener hasta 100 libros. En cuarto lugar, se observó que el promedio del nivel de instrucción materna fue de 4.8 lo que indica que la madre apenas concluyó la educación secundaria o bachillerato. Finalmente, al igual que en las variables anteriores, se observa que existe una gran variabilidad en el nivel de desempeño numérico de los niños. Así, existen niños que pudieron completar correctamente 53 ítems del test mientras que otros respondieron de manera acertada solamente 14 ítems. Más aún, los datos indican que, en promedio, los niños pudieron resolver apenas el 70\% del test, lo cual resulta sorprendente ya que el TENA fue desarrollado en base a las destrezas numéricas obligatorias 
del currículo nacional para primer año de básica por lo que se esperaría que los niños alcancen un mayor desempeño numérico.

\subsection{Asociación entre actividades numéricas, actividades de lectoescritura, nivel de instrucción materna y el desempeño numérico de los niños}

Para responder las cuatro primeras preguntas de investigación, esto es, si el desempeño numérico está asociado con actividades numéricas en el hogar, actividades de lectoescritura en el hogar, número de libros para niños, y/o el nivel de instrucción materna, en primer lugar se calculó la correlación de Spearman entre las diferentes variables bajo estudio. Los resultados de estos análisis se presentan en la Tabla 3.

Tabla 1. Media, rango y desviación estándar de las cinco variables bajo estudio.

\begin{tabular}{lcccc}
\hline \multicolumn{1}{c}{ Variables } & $M$ & $D E$ & Mínimo & Máximo \\
\hline Actividades numéricas & 1.99 & 0.79 & 0.29 & 3.76 \\
Actividades de lectoescritura & 2.26 & 0.99 & 0.00 & 4.00 \\
Número de libros para niños & 10.16 & 12.43 & 0.00 & 100.00 \\
Instrucción materna & 4.81 & 1.65 & 1.00 & 8.00 \\
TENA & 37.78 & 8.27 & 14.00 & 53.00 \\
\hline
\end{tabular}

Tabla 2. Media, desviación estándar y mediana de actividades numéricas y de lectoescritura.

\begin{tabular}{lcccc}
\hline \multicolumn{1}{c}{ Actividades numéricas } & $M$ & $D E$ & Mínimo & Máximo \\
\hline 1. Contar objetos & 3.22 & 1.13 & 0 & 4 \\
2. Clasificar objetos según el tamaño, color o forma & 2.64 & 1.32 & 0 & 4 \\
3. Contar de forma descendente & 1.69 & 1.44 & 0 & 4 \\
4. Escribir numerales & 3.17 & 1.08 & 0 & 4 \\
5. Identificar los nombres de los números & 2.54 & 1.31 & 0 & 4 \\
6. Unir números para formar figuras & 1.81 & 1.51 & 0 & 4 \\
7. Usar libros que tengan actividades con números & 2.34 & 1.36 & 0 & 4 \\
8. Leer cuentos numéricos & 1.72 & 1.44 & 0 & 4 \\
9. Jugar cartas & 1.93 & 1.50 & 0 & 4 \\
10. Hacer colecciones & 2.08 & 1.49 & 0 & 4 \\
11. Jugar juegos de tablero con dados & 1.53 & 1.44 & 0 & 4 \\
12. Ser tomado el tiempo & 1.99 & 1.51 & 0 & 4 \\
13. Hacer que su hijo use el reloj & 1.22 & 1.32 & 0 & 4 \\
14. Medir ingredientes mientras cocina & 1.36 & 1.36 & 0 & 4 \\
15. Utilizar el calendario & 1.11 & 1.22 & 0 & 4 \\
16. Hablar sobre dinero cuando están de compras & 2.10 & 1.53 & 0 & 4 \\
17. Jugar con calculadora & 1.57 & 1.43 & 0 & 4 \\
\hline \multicolumn{1}{c}{ Actividades de lectoescritura } & & & & \\
\hline 1. Escribir las letras & 2.74 & 1.27 & 0 & 4 \\
2. Identificar sonidos de las letras del alfabeto & 2.03 & 1.30 & 0 & 4 \\
3. Identificar nombres de las letras escritas del alfabeto & 2.01 & 1.33 & 0 & 4 \\
\hline
\end{tabular}


Tabla 3. Correlación de Spearman entre las variables bajo estudio.

\begin{tabular}{llcccc}
\hline \multicolumn{1}{c}{ Variables } & 1 & 2 & 3 & 4 & 5 \\
\hline 1 Actividades numéricas & 1 & $.65^{* *}$ & .14 & .02 & -.10 \\
2 Actividades de lectoescritura & & 1 & $.16^{*}$ & .07 & -.02 \\
3 Número de libros para niños & & & 1 & $.39^{* *}$ & $.23^{* *}$ \\
4 Instrucción materna & & & & 1 & $.28^{* *}$ \\
5 TENA & & & & & 1 \\
\hline
\end{tabular}

** La correlación es significativa en el nivel .01

* La correlación es significativa en el nivel .05

Como se puede observar, no existe una correlación significativa entre las experiencias numéricas en el hogar y el desempeño numérico de los niños. Tampoco hay una relación entre las experiencias de lectoescritura en el hogar y el desempeño numérico de los niños. Sin embargo, tanto el número de libros para niños en el hogar, como el nivel de instrucción materna, se correlacionan significativamente con el desempeño numérico. En otras palabras, la frecuencia con la que los padres realizan actividades numéricas y de lectoescritura junto con sus hijos no está relacionada con el desempeño numérico de sus hijos al finalizar el primer año de básica. No obstante, la presencia de libros para niños en casa, así como el nivel de instrucción materna, son factores que están asociados con el desempeño numérico de los niños. Existen dos datos que, aunque no forman parte de las preguntas de investigación valen la pena reportar. El primero es que las actividades numéricas están significativamente asociadas con las actividades de lectoescritura, lo que sugiere que los padres que más realizan actividades numéricas también realizan mayores actividades de lectoescritura con sus hijos. El segundo es que el número de libros en el hogar se relaciona positivamente con el nivel de instrucción materna. Este hecho sugiere que las madres más instruidas son las que más adquieren libros para sus hijos. También podría deberse a que el nivel de instrucción materna ha sido asociado al nivel socioeconómico (Aunio \& Niemvirta, 2010), i.e. mientras mayor el nivel de instrucción materna, mayor nivel socioeconómico; entonces, una mejor situación económica de la familia facilita un mayor acceso a libros.

Tabla 4. ANOVA para cortes en percentiles del número de libros para niños y el nivel de instrucción materna, respecto a TENA.

\begin{tabular}{|c|c|c|c|c|c|c|}
\hline & Equivalencia & $\mathrm{n}$ & Media TENA & $D E$ & $F$ & Post Hoc Tukey \\
\hline \multirow{4}{*}{$\begin{array}{l}\text { Número de libros } \\
\text { para niños }\end{array}$} & a. 4 libros & 63 & 35.29 & 8.02 & \multirow{4}{*}{$4.047 * *$} & $\mathrm{a}=\mathrm{b}, \mathrm{a}=\mathrm{c}, \mathrm{a} \neq \mathrm{d}$ \\
\hline & b. 6 libros & 31 & 37.52 & 8.94 & & $\mathrm{~b}=\mathrm{a}, \mathrm{b}=\mathrm{c}, \mathrm{b} \neq \mathrm{d}$ \\
\hline & c. 12 libros & 43 & 38.95 & 7.68 & & $c=a, c=b, c=d$ \\
\hline & d. 13 o más & 40 & 40.68 & 7.79 & & $\mathrm{~d} \neq \mathrm{a}, \mathrm{d} \neq \mathrm{b}, \mathrm{d}=\mathrm{c}$ \\
\hline \multirow{4}{*}{ Instrucción materna } & a. Primaria & 49 & 35.08 & 8.25 & \multirow{4}{*}{$4.942 * *$} & $a=b, a=c, a \neq d$ \\
\hline & b. Secundaria & 70 & 37.91 & 8.01 & & $\mathrm{~b}=\mathrm{a}, \mathrm{b}=\mathrm{c}, \mathrm{b}=\mathrm{d}$ \\
\hline & c. Tecnológica & 21 & 36.76 & 7.54 & & $\mathrm{c}=\mathrm{a}, \mathrm{c}=\mathrm{b}, \mathrm{c} \neq \mathrm{d}$ \\
\hline & d. Superior & 37 & 41.70 & 7.89 & & $\mathrm{~d} \neq \mathrm{a}, \mathrm{d}=\mathrm{b}, \mathrm{d} \neq \mathrm{c}$ \\
\hline
\end{tabular}

** La asociación es significativa en el nivel .01

Dadas las asociaciones significativas entre el número de libros para niños y el TENA, así como entre el nivel de instrucción materna y el TENA, se procedió a realizar cortes en los cuartiles de estas dos variables correlacionadas con el TENA y se realizó la comparación de cuartiles empleando la prueba ANOVA con análisis post hoc de Tukey. Los cortes permitieron establecer cuatro grupos para cada una de las variables (percentil 25, 50, 75 y percentil 100). Como se observa en la Tabla 4, el número de libros para niños presenta una asociación en el percentil 25 con el nivel más bajo de TENA. En general, a medida que aumenta el número de libros, aumenta el promedio en el TENA. La prueba post hoc de Tukey muestra que no hay diferencias significativas en el TENA entre poseer 0-4 libros, 5-6 libros y 712 libros; tampoco se advierte diferencias entre poseer 7-12 libros y 13 libros o más, sin embargo, sí 
hay diferencia entre poseer de 0 a 6 libros con respecto a poseer 13 libros o más. Ocurre algo similar con el nivel de instrucción materna, en el cual se advierte que el primer cuartil es justamente aquel que presenta un nivel de TENA más bajo. La prueba post hoc de Tukey en este caso muestra que los niveles de instrucción materna primaria, secundaria y tecnológica no evidencian diferencias significativas en el TENA; tampoco existen diferencias significativas entre los niveles de instrucción materna secundaria y superior, no obstante, sí se observan diferencias entre el nivel de instrucción primaria y tecnológica con respecto al nivel de instrucción superior.

Una vez realizados los cortes en los cuartiles del número de libros para niños y del nivel de instrucción materna, se realizó un análisis de regresión lineal múltiple con la finalidad de examinar si el número de libros para niños que las familias tienen en su hogar, así como el nivel de instrucción materna explican el desempeño numérico de los niños al finalizar el primer año de básica. Para ello, se introdujeron en el modelo el número de libros para niños y nivel de instrucción materna como variables independientes y el puntaje de los niños en el TENA como la variable dependiente. Como se resume en la Tabla 5, tanto el número de libros como el nivel de instrucción materna son predictores del desempeño numérico de los niños. Más específicamente, el modelo predice que por cada punto de incremento en el número de libros para niños en el hogar se incrementará en promedio 1.35 puntos en el TENA. Así mismo, por cada punto adicional en el nivel de instrucción materna, el modelo predice que en promedio aumentará 1.47 puntos en el TENA.

Tabla 5. Regresión lineal múltiple para predecir el puntaje de TENA al final del año lectivo.

\begin{tabular}{|c|c|c|c|c|c|c|}
\hline \multirow[t]{2}{*}{ Modelo } & & \multicolumn{2}{|c|}{$\begin{array}{l}\text { Coeficientes no } \\
\text { estandarizados }\end{array}$} & \multirow{2}{*}{$\begin{array}{c}\begin{array}{c}\text { Coeficientes } \\
\text { estandarizados }\end{array} \\
\text { Beta }\end{array}$} & \multirow[t]{2}{*}{$t$} & \multirow[t]{2}{*}{ Sig. } \\
\hline & & $B$ & Error estándar & & & \\
\hline \multirow{3}{*}{1} & Constante & 31.32 & 1.60 & & 19.56 & .00 \\
\hline & Libros para niños & 1.35 & 0.53 & .193 & 2.53 & .01 \\
\hline & Instrucción materna & 1.47 & 0.58 & .192 & 2.52 & .01 \\
\hline
\end{tabular}

Nota. $\mathrm{R}^{2}=.09$

Finalmente, para responder a nuestra quinta y última pregunta de investigación, es decir, si la frecuencia con la que los padres reportan realizar actividades numéricas y de lectoescritura en el hogar varía según el género de sus hijos, se realizó una prueba U de Mann-Whitney. Los resultados indican que no existe diferencia de género en la frecuencia de actividades numéricas y de lectoescritura que los padres realizan con sus hijos (actividades numéricas $U=3425.00, p=150$; actividades de lectoescritura $U=3872.00, p=899$ ). Es decir, estas actividades en el hogar se realizan de manera equitativa tanto con hijos varones como con mujeres.

\section{DISCUSIÓN}

El principal objetivo del presente estudio fue explorar la relación existente entre el ambiente de aprendizaje en el hogar y el nivel de instrucción materna con el desempeño numérico de los niños cuencanos al finalizar el primer año de básica. Para ello, se les pidió a los padres que completen un cuestionario en el que indiquen la frecuencia con la que realizan actividades numéricas y de lectoescritura con sus hijos y que señalen el número de libros para niños que tienen disponibles en el hogar. Adicionalmente, se recolectó información sobre el nivel de instrucción materna de los niños participantes.

Un primer hallazgo de este estudio es la gran variabilidad en la frecuencia con la que los padres reportan realizar actividades numéricas y de lectoescritura con sus hijos, lo cual coincide con lo observado en estudios previos (Lefevre et al., 2009; Missall et al., 2015; Skwarchuk, 2009). Así, mientras algunos padres reportaron realizar actividades numéricas y de lectoescritura a diario, otros indicaron que no las realizan nunca. En general, los bajos niveles de actividades reportados sugieren 
que la exposición a actividades numéricas y de lectoescritura ocurre de manera incidental y poco frecuente en los hogares cuencanos. Este hecho podría estar relacionado con la idiosincrasia cultural de los padres cuencanos. Al respecto, Ng \& Rao (2010), señalan que los valores culturales sobre la importancia de la matemática y sobre las expectativas de los padres acerca del aprendizaje de habilidades numéricas a temprana edad, están relacionados con la frecuencia de las prácticas educativas en el hogar. Un estudio comparativo demostró que los niños en países de Asia Oriental recibían más práctica e instrucción en aritmética que los niños en Europa y América del Norte (Ni, Chiu, \& Cheng, 2009). Entonces, podría ser que los padres cuencanos consideran que no es muy relevante que sus hijos realicen actividades numéricas y de lectoescritura en el hogar cuando son pequeños y por ello el número de actividades, relacionadas con estas habilidades, que realizan en sus hogares es poco frecuente. Finalmente, consistente con estudios previos (Lefevre et al., 2009; Skwarchuk, 2009), se observó que los padres cuencanos reportaron realizar actividades de lectoescritura más frecuentemente que actividades numéricas y reportaron realizarlas con la misma frecuencia tanto si se trataba de hijos varones como de mujeres. Estos resultados sugieren que tal vez los padres reciben mayor información sobre la importancia de la lectura que acerca de la importancia del número (Lefevre et al., 2009).

Un segundo hallazgo es la falta de correlación del reporte de los padres sobre la frecuencia de actividades numéricas y de lectoescritura que realizan en el hogar con el nivel de desempeño numérico de los niños. Este resultado concuerda con los resultados de estudios previos (Blevins-Knabe \& MusunMiller, 1996; Missall et al., 2015), sin embargo, también discrepa de otros estudios que evidenciaron que los reportes de los padres sobre las actividades numéricas realizadas en el hogar se correlacionaron con el desempeño numérico de sus hijos (LeFevre et al., 2010; Lefevre et al., 2009). Aunque no se sabe con exactitud el motivo de la inconsistencia entre los diversos estudios, incluyendo el presente estudio, se considera que los resultados aquí reportados podrían tener al menos tres explicaciones. La primera es que, posiblemente, los padres participantes fueron menos precisos en reportar sus actividades numéricas (Blevins-Knabe \& Musun-Miller, 1996) ya sea de manera involuntaria o de manera voluntaria, debido a la necesidad de aceptación social. Otra explicación podría ser que los niños cuencanos sí realizan actividades numéricas y de lectoescritura en el hogar, pero lo hacen con sus hermanos, y no precisamente con sus padres. Al respecto, la tasa promedio miembros de familia en Ecuador es de 3.9 lo que equivale a 1.9 hijos por hogar (INEC, 2012), siendo ésta mayor a otros países donde se han realizado estudios sobre las actividades numéricas y de lectoescritura en el hogar, como son Canadá y Holanda. Por ejemplo, en Canadá, país donde fue desarrollado el cuestionario empleado en el presente estudio, la tasa promedio de miembros de la familia es de 2.5, equivalente a 0.5 hijos por familia (STATCAN, 2007). Entonces, es evidente que en los hogares ecuatorianos existen al menos dos niños en casa que puede realizar conjuntamente actividades numéricas y de lectoescritura. Una última explicación podría ser que lo niños que asisten a centros educativos a temprana edad desarrollen mayores habilidades numéricas que los niños que no asisten a dichos centros, a pesar de tener ambientes de aprendizaje poco estimulantes en sus hogares. Lamentablemente, desconocemos si los niños del presente estudio asistieron a centros educativos antes de iniciar el primer año de básica por lo que no podríamos probar esta suposición. Estudios futuros que incluyan tanto niños que han recibido educación preescolar como a aquello que no la han recibido, son necesarios con el fin de determinar la influencia de la educación temprana en centros educativos en el desarrollo de las habilidades numéricas de los infantes. Por último, en el presente estudio se evaluó la frecuencia de actividades numéricas y de lectoescritura en el hogar, pero no la calidad de la interacción entre padres e hijos durante esas actividades. Sería importante que se investigue más a fondo qué hacen exactamente los padres durante una actividad numérica y de lectoescritura con sus hijos.

Como tercer hallazgo, se encontró que el número de libros para niños disponibles en el hogar, se relacionó positivamente con las habilidades numéricas de los niños, lo cual está en línea con resultados de estudios anteriores (Anderson, Anderson, \& Shapiro, 2005; Evans et al., 2014; Van den HeuvelPanhuizen \& Elia, 2012). Desafortunadamente, se observó que la cantidad de libros para niños difería ampliamente entre las familias cuencanas con familias que reportan tener hasta cien libros y otras que reportan no contar con ningún libro para sus hijos. Aún más, este estudio reveló que el número de libros en el hogar se relaciona positivamente con el nivel de instrucción materna, posiblemente, las madres más instruidas sean justamente las que compran más libros para sus hijos. Si consideramos las evidencias que indican que los libros benefician especialmente a niños de familias con poca educación 
y con empleos de baja remuneración (Evans et al., 2014), es necesario que las personas encargadas de las políticas educativas del país tomen decisiones urgentes que permitan el acceso a libros infantiles a los niños provenientes de familias de escasos recursos. Junto a ello, se hace necesario trabajar con las familias para que tomen conciencia de que los libros desempeñan un papel crucial en el desarrollo de las habilidades numéricas de sus hijos con el fin de que los libros sean parte integral del estilo de vida de estas familias.

Un cuarto y último hallazgo del presente estudio es que, al igual que en estudios anteriores (Anders et al., 2012; Aunio \& Niemivirta, 2010), el nivel de instrucción de la madre se relacionó positivamente con el desempeño numérico de los niños. Como se reportó anteriormente, el nivel de instrucción materna ha sido asociado con el nivel socioeconómico de la familia. Por ello, mejorar las competencias numéricas de los niños provenientes de niveles socioeconómicos bajos es de particular importancia no solo para ayudarlos a comenzar mejor la educación formal, sino también como un medio para reducir la brecha de rendimiento entre los niños pertenecientes a niveles socioeconómicos bajos y los niños de niveles socioeconómicos medios y altos (Jordan et al., 2009). Por lo tanto, se requieren programas de intervención que incluyan principalmente a niños de niveles socioeconómicos bajos con miras a mejorar sus habilidades numéricas. Estos programas deberían centrarse tanto en intervenciones efectivas dentro de la escuela como fuera de ella, es decir en los hogares (Starkey \& Klein, 2000).

Finalmente, la metodología utilizada en este estudio tiene al menos tres limitaciones. La primera es que el cuestionario empleado fue desarrollado en Canadá, ello implica que, eventualmente, los padres ecuatorianos realicen ciertas actividades con sus hijos, que no constan en el cuestionario empleado, por ejemplo "jugar con dados" o "escuchar canciones con números". No obstante, esta es solo una suposición que debería ser probada en estudios futuros que incluyan una mayor gama de actividades numéricas y de lectoescritura que puedan observarse en los hogares cuencanos. Una segunda limitación es que el reporte de los padres sobre las actividades realizadas en el hogar es subjetivo y puede no reflejar con exactitud su comportamiento real, lo cual podrían estar influenciado por un deseo de aceptación social. Por ello, a más del cuestionario se deberían emplear otros instrumentos que ayuden a recopilar con mayor precisión información sobre las experiencias en el hogar, por ejemplo, a través de observaciones en los hogares, o entrevistas a los niños. Por último, la tercera limitación tiene que ver con el número de preguntas acerca de las actividades numéricas y de lectoescritura presentes en el cuestionario empleado, el mismo que consta de 17 preguntas sobre el número y solamente tres sobre lectoescritura. Esto limita el tener un mayor entendimiento sobre el último tipo de actividades. Por lo tanto, se requiere equipar el número de preguntas empleadas para las actividades de lectoescritura y para las actividades numéricas en el hogar. Investigaciones futuras podría centrarse en ello.

\section{BIBLIOGRAFÍA}

Anders, Y., Grosse, C., Rossbach, H.-G., Ebert, S., Weinert, S. (2013). Preschool and primary school influences on the development of children's early numeracy skills between the ages of 3 and 7 years in Germany. School Effectiveness and School Improvement, 24(2), 195-211. https://doi.org/10.1080/09243453.2012.749794

Anders, Y., Rossbach, H. G., Weinert, S., Ebert, S., Kuger, S., Lehrl, S., von Maurice, J. (2012). Home and preschool learning environments and their relations to the development of early numeracy skills. Early Childhood Research Quarterly, 27(2), 231-244. https://doi.org/10.1016/j.ecresq.2011.08.003

Anderson, A., Anderson, J., Shapiro, J. (2005). Supporting multiple literacies: Parents' and children's mathematical talk. Mathematics Education Research Journal, 16(3), 5-26. https://doi.org/10.1007/BF03217399

Aunio, P., Niemivirta, M. (2010). Predicting children's mathematical performance in grade one by early numeracy. Learning and Individual Differences, 20(5), 427-435. https://doi.org/10.1016/j.lindif.2010.06.003

Clements, D. H., Sarama, J. (2007). Effects of a preschool mathematics curriculum: Summative 
research on the Building Blocks project. Journal for Research in Mathematics Education, 38(2), 136-163. https://doi.org/10.2307/30034954

Clements, D. H., Sarama, J. (2008). Experimental evaluation of the effects of a research-based preschool mathematics curriculum. American Educational Research Journal, 45(2), 443-494. https://doi.org/10.3102/0002831207312908

Clements, D. H., Sarama, J. (2011). Mathematics knowledge of low-income entering preschoolers. Far East Journal of Mathematical Education, 6, 41-63.

Duncan, G. J., Dowsett, C. J., Claessens, A., Magnuson, K., Huston, A. C., Klebanov, P., Pagani, L. S., Feinstein, L., Engel, M., Brooks-Gunn, J., Sexton, H., Duckworth, K., Japel, C. (2007). School readiness and later achievement. Developmental Psychology, 43(6), 1428-1446. https://doi.org/10.1037/0012-1649.43.6.1428; 10.1037/0012-1649.43.6.1428.supp (Supplemental)

Geary, D. C. (2011). Cognitive predictors of achievement growth in mathematics: a 5-year longitudinal study. Developmental Psychology, 47(6), 1539-52. https://doi.org/10.1037/a0025510

Geary, D. C., Hoard, M. K., Nugent, L. (2012). Independent contributions of the central executive, intelligence, and in-class attentive behavior to developmental change in the strategies used to solve addition problems. Journal of Experimental Child Psychology, 113(1), 49-65. https://doi.org/10.1016/j.jecp.2012.03.003

INEC (2012). Encuesta nacional de ingresos y gastos. Quito, Ecuador. Recuperado a partir de: www.ecuadorencifras.gob.ec/documentos/webinec/Estadisticas_Sociales/Encuesta_Nac_Ingresos_Gastos_Hogares_Urb_Rur_ENIGHU/ENIG HU-2011-2012/EnighurPresentacionRP.pdf

Jordan, N. C., Kaplan, D., Ramineni, C., Locuniak, M. N. (2009). Early math matters: Kindergarten number competence and later mathematics outcomes. Developmental Psychology, 45(3), 850-867. https://doi.org/10.1037/a0014939.

Krajewski, K., Schneider, W. (2009). Early development of quantity to number-word linkage as a precursor of mathematical school achievement and mathematical difficulties: Findings from a four-year longitudinal study. Learning and Instruction, 19(6), 513-526. https://doi.org/10.1016/j.learninstruc.2008.10.002

LeFevre, J.-A., Polyzoi, E., Skwarchuk, S.-L., Fast, L., Sowinski, C. (2010). Do home numeracy and literacy practices of Greek and Canadian parents predict the numeracy skills of kindergarten children? International Journal of Early Years Education, 18(1), 55-70. https://doi.org/10.1080/09669761003693926

Lefevre, J. A., Kwarchuk, S. L., Smith-Chant, B. L., Fast, L., Kamawar, D., Bisanz, J. (2009). Home numeracy experiences and children's math performance in the early school years. Canadian Journal of Behavioural Science, 41(2), 55-66. https://doi.org/10.1037/a0014532

Locuniak, M. N., Jordan, N. C., Kaplan, D., Ola, L. N. (2006). Number sense growth in kindergarten: A longitudinal investigation of children at risk for mathematics difficulties, 77(1), 153-175.

Melhuish, E., Phan, M., Sylva, K., Sammons, P., Siraj-Blatchford, I., Taggart, B. (2008). Effects of the home learning environment and preschool center experience upon literacy and numeracy development in early primary school. Journal of Social Issues, 64(1), 95-114. https://doi.org/10.1111/j.1540-4560.2008.00550.x

Missall, K., Hojnoski, R. L., Caskie, G. I. L., Repasky, P. (2015). Home numeracy environments of preschoolers: Examining relations among mathematical activities, parent mathematical beliefs, and early mathematical skills. Early Education and Development, 26(3). https://doi.org/10.1080/10409289.2015.968243

Pedhazur, E. J. (1997). Multiple regression in behavioral research: Explanation and prediction (3rd ed.). Fort Worth, TX: Harcourt Brace College Publishers.

Ramani, G. B., Siegler, R. S. (2011). Reducing the gap in numerical knowledge between low- and middle-income preschoolers. Journal of Applied Developmental Psychology, 32(3), 146-159. 
https://doi.org/10.1016/j.appdev.2011.02.005

Rendón-Macías, M., Villasís-Keever, M., \& Miranda-Novales, M. (2016). Estadística descriptiva. Rev Alerg Mex, 63(4), 397-407.

Siegler, R. S., Ramani, G. B. (2008). Playing linear numerical board games promotes low-income children's numerical development. Developmental Science, 11(5), 655-661. https://doi.org/10.1111/j.1467-7687.2008.00714.x

Siegler, R. S., Ramani, G. B. (2009). Playing linear number board games - but not circular ones improves low-income preschoolers' numerical understanding. Journal of Educational Psychology, 101(3), 545-560. https://doi.org/10.1037/a0014239

Skwarchuk, S. L. (2009). How do parents support preschoolers' numeracy learning experiences at home? Early Childhood Education Journal, 37(3), 189-197. https://doi.org/10.1007/s10643-0090340-1

Starkey, P., Klein, A. (2000). Fostering parental support for children's mathematical development: An intervention with head start families. Early Education and Development, 11(5), 659-680. https://doi.org/10.1207/s15566935eed1105

Starkey, P., Klein, A., Wakeley, A. (2004). Enhancing young children's mathematical knowledge through a pre-kindergarten mathematics intervention. Early Childhood Research Quarterly, 19(1), 99-120. https://doi.org/10.1016/j.ecresq.2004.01.002

STATCAN (2007). Census families, number and averages size. Recuperado a partir de www.statcan.gc.ca/tables-tableaux/sum-som/101/cst01/famil40-eng.htm

Swanson, H. L., Jerman, O., Zheng, X. (2008). Growth in working memory and mathematical problem solving in children at risk and not at risk for serious math difficulties. Journal of Educational Psychology, 100(2), 343-379. https://doi.org/10.1037/0022-0663.100.2.343

van den Heuvel-Panhuizen, M., Elia, I. (2012). Developing a framework for the evaluation of picturebooks that support kindergartners' learning of mathematics. Research in Mathematics Education, 14(1), 17-47. https://doi.org/10.1080/14794802.2012.657437 